\title{
Cost effectiveness and quality of life considerations in the treatment of hepatitis $C$ infection
}

This article was published in the following Dove Press journal:

ClinicoEconomics and Outcomes Research

13 July 2010

Number of times this article has been viewed

\section{$\mathrm{H}$ Jafferbhoy \\ W Gashau \\ JF Dillon}

Gut Group, Biomedical Research Institute, University of Dundee, Ninewells Hospital and Medical School, Dundee, UK
Correspondence: John F Dillon

Gut Group, Level 5, Biomedical Research Institute, University of Dundee,

Ninewells Hospital and Medical School, Dundee, UK DDI 9SY

Tel +44 I382660 I I I

Email j.f.dillon@dundee.ac.uk.

\begin{abstract}
Chronic hepatitis $\mathrm{C}^{1}$ is a common cause of liver disease worldwide. It is a slow and progressive condition which can lead to decompensated cirrhosis and hepatocellular carcinoma. Hepatitis $\mathrm{C}$ virus ${ }^{1}$ impairs quality of life (QOL) even in the absence of chronic liver disease, but its relative silent nature can lead to a delay in diagnosis. The current standard of care of treatment is pegylated interferon and ribavarin. This achieves a sustained virological response (SVR), which is a cure of infection, in up to $80 \%$ of patients depending on viral genotype. The attainment of SVR improves survival, avoids long-term complications, and improves QOL. But treatment is not only expensive; there are issues of tolerability and adverse effects. This has led to a multitude of cost effective analysis and health technology assessment on HCV treatment. This overview discusses the natural history of the virus infection and its effect on the patients' QOL. It focuses on the treatment options available, their efficacy, and cost effectiveness. It reviews the evaluations that suggest combination therapy is cost effective and explores the assumptions and limitations of these studies in real world treatment arenas.
\end{abstract}

Keywords: hepatitis C, quality of life, cost effective

\section{Introduction}

The liver is the largest organ in the body and performs a host of functions including the processing and storage of nutrients and vitamins, degradation or elimination of metabolites, and the synthesis of plasma proteins including coagulation factors. Its ability to regenerate makes it unique and robust allowing it to survive various insults without sustained loss of function or capacity. Hepatitis C virus ${ }^{1}$-related viral hepatitis is one of the causes of hepatic injury resulting initially in inflammation, which if persistent and protracted, can cause fibrosis leading to cirrhosis. Hepatitis $\mathrm{C}$ infection is rapidly becoming one of the leading threats to public health internationally as a cause of current and future cirrhosis. National treatment plans are being developed, and it is vital given the size of this epidemic that these plans are based on robust evidence of cost-effectiveness.

\section{Hepatitis $C$ virus: the background}

$\mathrm{HCV}$ is an enveloped, single-stranded RNA virus that causes acute or chronic infection. Transmission of the virus is parenteral in nature with needle sharing, unscreened blood transfusions, non-sterile tattooing or acupuncture, vertical and sexual transmission being among some of the means of viral exposure.

11 genotypes of the virus have been identified of which 6 predominate internationally. There are clear differences in the distribution of the genotypes around the globe. Worldwide, genotypes 1-3 are most prevalent. Genotype $1 \mathrm{a}$ and $1 \mathrm{~b}$ account for $60 \%$ 
of all the infections globally. It is most prevalent in Europe, North America, and Japan. Genotypes 2 and 3 are seen in these countries less often and usually represent the minority of infections. Genotype 3 is endemic in south-east Asia. Genotype 4 is principally found in the Middle East, Egypt, and central Africa. Genotype 5 is almost exclusively found in South Africa. Of the remaining, genotype 6 is the most prevalent and along with more minor genotypes numbered 7-11 are seen in various countries in Asia. ${ }^{2}$

Within the UK, genotype 1 (G1) is most common, accounting for $40 \%-50 \%$ of infected cases, most commonly in those infected through blood products. ${ }^{3}$ The second largest group is genotypes 2 and 3, most commonly seen among drug users. In the UK, genotype 3 predominates over genotype 2 . Genotypes 4-6 account for 5\% of the infected subgroup and are more prevalent in other parts of the world.

Intravenous drug use remains the most common cause of $\mathrm{HCV}$ infection by a large margin and is the pre-eminent risk factor for future infection, with an estimated $50 \%$ prevalence of HCV infection among intravenous drug users. ${ }^{3,4}$ In many cohorts of patients, especially in developed countries, more than $70 \%$ have acquired infection by this route. In approximately $10 \%-40 \%$ of individuals the risk factor is never identified.

Globally, HCV infection affects $3 \%$ of the population with an estimated 170 million being chronic carriers. ${ }^{2}$ Within the UK, the estimated prevalence is approximately 185,000 , about $0.5 \%$ of the population. In Scotland, approximately 50,000 are estimated to be infected, with 37,500-39,000 of those infected chronically, nearly $1 \%$ of the population. ${ }^{4}$ Over three-fifths of those with chronic HCV infection in Scotland are male, with $88 \%$ of those diagnosed being under 50 years of age. This is very much in keeping with demographics internationally. Infection is thought to be more common in men for two reasons: men are more likely to inject drugs, and there are also men affected by hemophilia and more likely to have been infected with blood products. ${ }^{5}$

Acute infection is often asymptomatic and rarely results in hepatic failure. Of those infected, 70\%-90\% become chronic carriers of the virus. ${ }^{6}$ The natural history of chronic hepatitis is ill defined due to the long latent period between inoculum and development. Severity and progression is variable but generally slow. ${ }^{7}$

Complications of chronic hepatitis $\mathrm{C}(\mathrm{CHC})$ include cirrhosis, hepatocellular carcinoma and decompensated liver failure most commonly manifesting as ascites and varices. Studies have looked prospectively, retrospectively, and in nonconcurrent prospective ways at the natural history of hepatitis $\mathrm{C}$. The differences between these approaches seem to show divergent outcomes, which have implications for the design of models of cost effectiveness.

The prospective studies looked mainly at the transfusionrelated NANB hepatitis and concluded that only $7 \%-15 \%$ developed cirrhosis and 1.3\%-3.7\% had liver-related death and even a smaller proportion progressed to HCC; however, follow-up on these studies, while long for prospective studies at between 15 and 25 years is short for the natural history of $\mathrm{HCV}^{8-11}$ In addition, receiving a blood transfusion is not a benign intervention, and many patients will have succumbed to the underlying disease that necessitated the transfusion and will thus have not expressed the natural history of HCV. This is further exacerbated by the average age of transfusion recipients, which again precludes the expression of the full natural history of HCV. Therefore estimates of rates of progression to HCV disease endpoints in these studies should be regarded as the low end of the range. The retrospective studies used a variety of strategies to identify their base population; all are biased, as the patients have been diagnosed for some reason, often because they have presented with a complication of HCV disease or have been collected in referral centers. Unsurprisingly these were on the other end of the spectrum reporting a higher rate of cirrhosis, ranging from $17 \%-55 \%$ and liver-related mortality of up to $15.3 \%$. The HCC incidence varied from $2 \%-23 \%$ depending on follow-up. ${ }^{12-15}$ The mixed retrospective prospective studies are again limited by the biases discussed previously. These reported cirrhosis in $0.3 \%-5.9 \%{ }^{16,17}$ but even higher at $15 \%$ in studies where follow-up was on men and longer than 40 years with development of HCC seen in $1.9 \% .{ }^{18}$ Liver-related mortality was reported between $1.0 \%$ and $2.1 \% .^{17}$

The differences reported in outcome and natural history is partly because of the groups studied and the methods employed to evaluate, as discussed above; although, host and environmental factors play a vital role in disease progression. Thus it is possible to view $\mathrm{HCV}$ natural history or progression as 3 groups; those that progress to cirrhosis within 20 years, those that progress over 20-50 years, and those that do not progress within their lifetimes. To which group an individual falls appears to depend more on host and environmental factors than purely viral factors. Invariable host factors that are associated with faster rates of fibrosis progression include advancing age, older age at infection, male gender, and high viral load. ${ }^{19}$ Genotype and ethnicity have not been firmly associated with disease progression but rather with reduced responsiveness to therapy, especially G1 and African Americans. ${ }^{20}$ The most significant variable associated with disease progression is alcohol consumption, 
even at levels only moderately raised. ${ }^{21,22}$ The obesity-related metabolic syndrome and its complex relation with fibrosis are increasingly being recognized as a contributing factor to fibrosis progression. ${ }^{23}$ There is gathering evidence that use of cannabis and tobacco is also associated with fibrosis.

More patients are living longer with HIV infection than ever before, and a significant number are co-infected with $\mathrm{HCV}$. Such individuals have a 2-fold increase in frequency and rate of development of cirrhosis; death from complications of liver disease now being one of the commonest causes of death among HIV-infected patients. ${ }^{24,25}$ These cofactors for progression have not been adjusted for in many models of disease progression. Once fibrosis is established and decompensation occurs, significant increases in morbidity and mortality result with only $51 \%$ surviving at 5 years compared with $91 \%$ of those with compensated cirrhosis. ${ }^{7,26}$ As a result of the disease process, HCV infection is now the most common cause of liver transplant within the developed world. ${ }^{27}$

It is expected that the number of those with complications secondary to this condition will rise over the coming decades. Aside from the increased burden on health care, many of those infected are young and will have a significantly reduced quality of life (QOL) without intervention. ${ }^{28}$ Consequently, there has been an increasing shift toward screening, diagnosing, and treating people with this condition.

\section{Treatment of hepatitis C}

The aim behind all treatment options is to eradicate HCV viremia thereby increasing QOL and reducing the risk of cirrhosis and hepatocellular carcinoma. Non-pharmacological treatment includes counseling at diagnosis and the management of non-specific symptoms such as fatigue. However pharmacological treatment is central as it can cure this chronic viral infection. Studies have confirmed and defined a surrogate marker for virlogical cure the absence of viral RNA in the patient's blood 6 months after the cessation of therapy, and is termed sustained virological response (SVR). All patients with $\mathrm{CHC}$ should be considered for combination therapy except those with absolute contraindications to treatment. ${ }^{29-31}$ Though most individuals with active drug misuse are not considered for therapy as it is assumed that they have poor compliance and higher incidence of side effects and risk of subsequent reinfection. ${ }^{32}$

Interferons (pegylated and non-pegylated) are the mainstay of treatment; they are normally used in conjunction with ribavarin. The mechanism of action is unclear but may include immune modulation at a cellular level as well as direct antiviral activity. Alpha interferon, both native and pegylated, result in flu-like symptoms, hematological abnormalities including neutropenia, anemia, and thrombocytopenia, and neuropsychiatric disturbances like depression, fatigue and suicidal ideation. Pegylated interferons have added polyethylene glycol molecules to delay renal clearance meaning the dose is delivered once weekly, which may have positive implications relating to compliance. Ribavarin is a purine nucleoside analogue and can be given weight based or a flat dose. Its major side effect is hemolytic anemia which leads to dose reduction or stoppage of therapy in $9 \%$ of patients. Patients receiving a dose higher than $1 \mathrm{gm} / \mathrm{d}$ are at risk of hemolytic anemia. ${ }^{33}$ Though a degree of anemia produced as a side effect in patients has been shown to be associated with higher viral clearance. Success of treatment has been shown to be influenced by 3 major factors: HCV genotype, high pre-treatment viral load, and increased fibrosis. In general, genotypes 1 and 4 respond less well to treatment compared with other genotypes. In order to maximize the adherence to optimal treatment regimen hematopoietic growth factors have been used. Erythropoietin has been used in patients who develop anemia $(<120 \mathrm{~g} / \mathrm{L})$ while on combination therapy (PEG interferon and ribavarin). It has been shown to improve anemia and consequently improve QOL. ${ }^{34,35}$ But to date it has yet to be demonstrated that this leads to an improvement in SVR or that this is cost effective. ${ }^{36}$

\section{Interferon monotherapy versus dual therapy}

The story of treatment starts with interferon alpha, which was shown to be beneficial in 1986 though monotherapy of varying length had limited success. In the late 1990s, 2 large randomized controlled trials assessed SVR comparing interferon monotherapy against the alternative of interferon plus ribavarin. ${ }^{37,38}$ Both trials demonstrated the superiority of combination therapy over monotherapy resulting in a significantly increased SVR. Generally, the use of dual therapy improved SVR when used for 12 months rather than 6 months, especially in patients with G1.

The results of these trials were further validated by meta-analyses of available studies, which reported SVR of $32 \%-41 \%$ with dual therapy compared with $8 \%-16 \%$ with monotherapy. ${ }^{39}$ As a result, dual therapy became the treatment of choice in $\mathrm{CHC}$ infection with clinical guidelines suggesting it be used in patients over 18 years of age with moderate to severe $\mathrm{HCV}$ infection for 6 months in treatment naïve patients with genotype 2 or 3 virus and 12 months in patients with G1 who have demonstrated a response by week 24 of treatment. ${ }^{31}$ 


\section{Dual therapy with peginterferon versus interferon}

The current standard of care is a combination of weekly subcutaneous injection of long-acting pegylated interferon and daily oral ribavarin. ${ }^{29,30}$ There are two commercially available licensed pegylated interferons: peginterferon alpha- $2 a$ and peginterferon alfa- $2 b$. They differ slightly in the interferon molecule by a few amino acids and in the size and nature of the covalently attached polyethylene glycol (PEG) moiety, with resulting differences in pharmacokinetics and in dosing regimens. ${ }^{40,41}$ Peginterferon alpha-2a has a very restricted volume of distribution, longer half-life, and reduced clearance compared with native interferon alpha-2a, and can be given once weekly independently of bodyweight. Peginterferon alpha- $2 b$ has a shorter half-life in serum than peginterferon alpha-2a and requires bodyweight-based dosing.

Two double-blind randomized trials compared the efficacy of dual therapy with ribavarin and pegylated or non-pegylated interferon. ${ }^{42,43}$ In the first trial PEG + ribavirin was compared with interferon + ribavirin, ${ }^{43}$ and in the second trial both these options were compared with PEG monotherapy ${ }^{43}$ It could be argued that the first trial is confounded as the benefits could be attributed to ribavirin, present in both treatment groups. However, ribavirin monotherapy has been shown to have little or no sustained virologic response. Furthermore, the results of the trial have since been externally validated by other studies.

At the end of 48 weeks treatment, both studies found a significant difference between dual therapies with PEG compared with non-PEG interferon. This difference was maintained at the end of the follow-up period where $54 \%-56 \%$ of patients had a sustained response compared with $44 \%-47 \%$ in the non-PEG dual therapy arm. The results of these studies were pooled and analyzed according to response. Genotypes 2 and 3 were identified as the most responsive to dual therapy, reaching SVR rates of $76 \%-82 \%$ at the end of follow-up. ${ }^{44}$ Subsequent studies have shown that HCV genotypes 2 and 3 can be treated with only 6 months of therapy with higher SVR. ${ }^{42}$ Davis also identified that virtually no patients with G1 went on to mount an SVR if at 12 weeks into dual therapy there was little or no reduction in the viral load. As a result of the data, it is now recommended that G1 patients with no early virologic response (EVR), defined as at least a two log drop in viral load, at 12 weeks should discontinue treatment. Patients with G1 who achieve EVR but are still HCV RNA positive should continue on treatment for 24 weeks. If PCR negative at this stage they should continue the full course of treatment; those positive should stop as they have no chance of an SVR. Those patients negative for HCV PCR at 12 weeks should have a full 48 weeks treatment. ${ }^{30}$

Criteria for participant inclusion and exclusion were similar in these trials. All included patients were treatment naive with liver biopsy within the last year. The majority of included patients were male, in their 40s, with mainly G1 and no significant co-morbidities. No explicit mention was made as to what proportion of the participants were drug users, former active or on opiate substitution therapy. It can be speculated that virtually no active substance users were included in the trials and that the proportions of former drug users in trials would be under represented. Most trials have excluded patients with co-infection with HIV, the rationale being that patients with a primary immune condition may not be able to mount a suitable response to result in successful and sustained virologic response. ${ }^{38,42,45}$ Additionally, progression of cirrhosis is increased by co-infection with HIV, which also makes viral clearance more difficult. The efficacy of therapy is reduced in this group, being dependent on the quality of HIV care and CD4 counts. ${ }^{46,47}$

Retrospective analysis of 4 studies considered the issues of compliance and adherence by dividing the participants into 2 broad groups: those with $>80 \%$ of the prescribed dose $80 \%$ of the time and those who were not as compliant. The study found that regardless of genotype, participants with high levels of adherence were more likely to have a sustained response at the end of follow-up. Most patients in the trials were adherent to their treatment regime over $80 \%$ of the time. ${ }^{48}$

Recently head to head studies between the two pegylated interferons have been reported, the largest being the IDEAL study reported no difference in SVR between the two drugs. ${ }^{49}$ The meta analysis by Awad et al which is statistically under powered suggests "that peginterferon alpha-2a is associated with higher SVR than peginterferon alfa- $2 b$. However, the paucity of evidence on adverse events curbs the decision to definitively recommend one peginterferon over the other because any potential benefit must outweigh the risk of harm". ${ }^{50}$ The size of advantage was approximately a $10 \%$ increase in SVR, if this was born out in full analysis then it would have a limited impact on cost-effectiveness analysis but one financially significant given the size of the HCV epidemic facing many health care providers.

\section{Quality of life issues}

As many patients are asymptomatic, the health service burden due to $\mathrm{HCV}$ infection is initially minimal. This is altered once cirrhosis is established. Once symptoms occur they tend to be non-specific in nature with fatigue being the 
most common symptom. ${ }^{51}$ Both patient and observer reported measures have been used to assess health-related quality of life ( HRQOL), disease impact, and mental health status in patients with $\mathrm{CHC}$. One such tool is SF-36 which is a validated short form health survey. Patients with $\mathrm{CHC}$ show deficiency particularly in the physical and general health scales in SF-36 as compared with a normal population. ${ }^{52}$ A limitation to this and other such tools is that measuring general health may fail to detect small but meaningful disease specific changes in QOL. Hence a Chronic Liver Disease Questionnaire(CLDQ) has been used to assess patients and is superior in measuring deterioration of QOL in patients with severe liver disease and CHC. ${ }^{53}$

There is an increasing body of evidence to show that QOL is reduced in HCV infection; however, manifesting symptoms are often nonspecific, or related to stigmatization, depression, and cognitive impairment. ${ }^{54}$

Studies objectively assessing QOL including vitality, ability to function socially, pain, and mood demonstrated reduced QOL measures in patients with hepatitis $\mathrm{C}$ similar to those in patients with Type 2 diabetes. Chronic hepatitis $\mathrm{C}$ carriers experienced more pain, less vitality, and reduced social functioning. ${ }^{55,56}$ One study confirmed reduced QOL measures among those with no history of drug use or chronic hepatitis infection; a subgroup that had previously been perceived to have little or no impact to their lives as a result of diagnosis. ${ }^{28}$ Increasing evidence also suggests mild cognitive decline in those with mild $\mathrm{CHC}$, which may also account for a decrease in QOL. ${ }^{57}$

Successful and sustained responses to treatment have been shown to improve life quality; ${ }^{55}$ this is further increased in those who receive dual therapy. ${ }^{52,58}$ Conversely, there is an increased emotional toll among those who are not selected for treatment with $71 \%$ of this group experiencing active psychiatric sequalae such as depression and psychosis, of whom $28 \%-40 \%$ may require treatment. ${ }^{59}$

Side effects of interferon therapy can be hard to tolerate. Injections can vary from once to thrice weekly depending on whether the pegylated or non-pegylated form is being used. Influenza-like symptoms are common after dosing. 50\% of patients suffer non-specific symptoms such as fatigue, arthralgia, weakness, headaches, pyrexia, myalgia, nausea, and insomnia. A quarter of those treated are affected by hair loss, mood changes, pruritis, dermatitis, and rigors. ${ }^{60}$ Starting and maintaining treatment over the course of 6-12 months can result in substantial costs that are intangible and difficult to measure. Other costs that are not routinely considered in literature include indirect costs such as lost productivity.

\section{Cost effectiveness of treatment of hepatitis C}

Several studies have attempted to assess the costs related to hepatitis $\mathrm{C}$. The full cost is sizeable predominantly because it is a condition that affects young adults. Due to the long latent period, many of the cost-effectiveness studies are based on hypothetical models that are updated when new data on clinical effectiveness is made available. As the standard of treatment is the use of pegylated interferon with ribavirin, this will remain the focus of this review of cost-effectiveness studies.

Bernfort et al considered cost effectiveness from the perspective of the Swedish health service in $2005^{61}$ using data from the Manns ${ }^{43}$ study in a Markov model, which allowed simulation of a hypothetical cohort of patients with hepatitis $\mathrm{C}$. The model was adapted to compare pegylated interferon ( $1.5 \mu \mathrm{g} / \mathrm{kg} /$ week) and ribavirin ( $800 \mathrm{mg}$ daily) with interferon (3MIU 3/week) and ribavirin (100 mg-1200 mg daily). The model included not only SVR rates over a period of 20 years but also a stopping rule: those with no early viral response, defined as a reduction in viral load of $2 \log$ units, were discontinued from treatment based on data from a study by Davis. Probabilities of transition from one health state to another over the defined time were based on studies on previous studies of Swedish patients with untreated HCV infection. Health states included cleared virus, moderate hepatitis, decompensated liver disease, hepatic encephalopathy, hepatocellular carcinoma, and death. By the end of the model, all patients were dead. The QOL measures and the weighting given to each measure were derived or influenced from earlier studies. ${ }^{62,63}$ Cost data were from the Swedish health care system, were considered fixed, and therefore not included into sensitivity analysis. All prices were expressed in 2005 Euros. Cost-effectiveness analysis was estimated using incremental cost-effectiveness ratios (ICER). Results were presented separately according to genotype. While it is difficult to transfer studies from other countries to the UK, the discounted incremental costs per quality associated life years (QALY) are presented here (approximate 2005 exchange rate $\left.£ 1=€ 1.46^{1}\right)$. For G1 patients, dual PEG + fixed ribavirin in comparison with dual IFN + ribavirin saved $£ 140$ with a gain of 0.29 QALYs, which makes PEG clearly dominant compared with interferon. For non-G1 patients, there are increased costs of $£ 645$ with a gain 0.09 QALY creating an incremental cost-effectiveness of $£ 7,161 /$ QUALY gained. Using an assumed willingness-to-pay (WTP), a cost of up to $€ 40,000$ (£27,397) for a QALY, the study demonstrated that PEG is cost effective even at very low WTP levels. Although 
treatment with PEG is cost effective from a health system point of view, when looked at from the assumed societal perspective, the cost-effectiveness was reduced even at high WTP values. However, since the study considered no costs or benefits from a societal viewpoint, it is hard to assess how applicable this is in reality. The study concluded that while PEG is clearly indicated in patients with $\mathrm{HCV}-1$ infection, it might not be as cost effective in HCV-2 or 3.

Siebert et $\mathrm{al}^{62}$ addressed the issue of cost effectiveness using data from the Manns ${ }^{43}$ study. Sustained virologic response rates were projected from each trial arm into a hypothetical Markov model. QOL measures were from a survey of German patients with $\mathrm{CHC}$. Mulitvariate regressional analysis was used to weigh each of the QOL measures. A panel of experts and a European QOL instrument was used to create the estimates used in the sensitivity analysis. Costs were from the German health care system and inflated and altered to match current rates. Cost effectiveness was measured in ICER with the perspective of society. Results were presented separately for fixed and weight-based dosing for ribavirin, as the trial identified a significant link between SVR and weight-based ribavirin dosing. Again, prices are presented here in Sterling (based on $£ 1=€ 1.95$ ). Dual therapy with PEG and weight-based ribavirin in comparison with interferon and ribavirin had an incremental cost per QALY of $£ 3,304$. This increased to $£ 6,051$ per QALY when ribavirin dose was fixed, suggesting weight-based ribvirin dosing is more cost effective than fixed doses, assuming all patients respond well to doses related to their weight. Further sensitivity analysis demonstrated that these incremental costutility values remained the best use of resources in clinical subgroups apart from those with genotype-2 HCV or high viral loads. It was speculated that this could be because of the high SVR achieved by the former of these groups and the low rates by the later irrespective of treatment.

Buti and colleagues ${ }^{64}$ reviewed not just effectiveness according to incremental cost but also benefits from the Spanish health system perspective. Patient characteristics and virologic response were obtained from Manns' work. The focus was on response according to genotype, the effect of dosing according to weight, and the effects of compliance with therapy. Costs came from the Spanish health care health system and did not consider production or indirect costs. The study investigators adjusted costs to current values of the year 2000. A discount rate of 3\% was applied to future benefits and costs. Using similar models and data as the previous studies, the results provided external validation of the findings of Siebert and other cost-effectiveness studies, suggesting that the use of peginterferon for hepatitis $\mathrm{C}$ treatment was superior for all patients. Focusing on G1 HCV-infected patients, peginterferon and fixed-dose ribavirin resulted in an incremental cost-effectiveness ratio of $£ 1,750$ per QALY gain. When ribavirin is adjusted to weight, this reduced the ICER to $£ 1,732$ and further dropped the cost to $£ 277$ per QUALY gain when patients were compliant with $80 \%$ of treatment over $80 \%$ of the treatment duration. The results demonstrated that the optimal treatment regime requires individual adjustment of ribavirin to patient weight and patient compliance. This increase in effectiveness is even more pronounced in the harder to treat patients with G1 infection. The study did not look at the cost effectiveness of stopping therapy early if no viral response is achieved in the first 6 months of treatment.

Shepherd et $\mathrm{al}^{65}$ assessed cost effectiveness of peginterferon and ribavarin in 2005. They used a similar hypothetical model to simulate the outcomes of a cohort of HCV patients. Unlike previous models, the age of diagnosis was younger at 36 years rather than 43 . Disease progression in the model was based on rates in literature with SVR rates being based on a meta-analysis of two large randomized controlled trials, ${ }^{43,44}$ while subgroup analysis was based on the study by Davis. Values relating to utility were taken from literature or estimated by a panel of experts using a standardized QOL scale. Costs were direct but excluded capital and overhead costs. Market value drug costs were summarized from the national formulary. Variations in SVR, discounting for both benefits and cost, and drug costs were addressed in a sensitivity analysis. As in previous studies, Shepherd and his colleagues confirmed the cost effectiveness of peginterferon and ribavirin over interferon alone at a cost of $£ 12,123 / \mathrm{QALY}$ gained. Predictably, the incremental cost-effectiveness ratio was increased in patients with a high viral load irrespective of genotype. When grading cost effectiveness by sustained response according to genotype, it was clear that $\mathrm{HCV}-2$ and -3 patients had the better estimates based on the outcomes of one study ( $£ 7,051 /$ QUALY saved) compared with the higher cost of $\mathrm{HCV}-1$ patients at $£ 10,848$. When SVR rates from the Manns study were used, the costs for $\mathrm{HCV}-2$ and -3 treatment rose to $£ 37,578$ /QUALY gain. The authors speculated that this might have been due to the marginal difference between the SVR rates of those treated with PEG versus interferon ( $82 \%$ and $79 \%$ respectively). This increase might be because of the increased response to treatment rate in this genotype subgroup. Even with variations of costs, discount, and SVR, the cost per QALY remained under $£ 30,000$, making PEG the most clinically and cost-effective 
pharmacological option. Additionally, the authors showed clearly that stopping treatment after 12 weeks in HCV-1 patients with no early virologic response could result in substantial cost savings of approximately $16 \%$. Were treatment to continue after the 12-week point in non-responders, the continuing cost of treatment would be $£ 226,573$ per QALY compared with the alternative of no treatment.

Several studies have assessed the cost effectiveness of peginterferon and ribavirin versus interferon and ribavirin therapy using hypothetical models to predict cost-utility ratios. Overall, the studies show dual treatment with pegylated interferon is superior to the use of interferon dual therapy, both clinically and cost-benefit wise. Results also demonstrate that genotypes 2 and 3 had lower ICER within subgroup analysis compared with G1 patients. Stopping treatment in patients with no early virologic response could save the health system a substantial amount in terms of cost and would also prevent further unnecessary side effects among this patient group.

\section{Cost effectiveness in real world settings}

In the main, the models described above have made realistic assumptions on the natural history of $\mathrm{HCV}$ infection and have used the best treatment outcome data available from the large randomized controlled trials. However these trials under represent key subgroups of patients for treatment: most significantly those on opiate substitution therapy and those who have recently given up intravenous drug use, which many authorities now regard as suitable for therapy. The cohort of patients being treated in many centers is primarily made up of people with concurrent issues such as drug abuse, but also including worries about accommodation, transport costs, and social care issues. Motivation for treatment is often reduced, as HCV infection is one of many life issues they have to face and is the one that is least pressing in the short term. This will clearly have an impact on compliance with therapy, which as has been shown above is a key determinant to achieving SVR. Little comment is passed regarding the number of included participants who are on a substance misuse stabilization program in the controlled trials that so many of the cost-effectiveness studies are extrapolated from. Sheerin and colleagues ${ }^{66}$ are one of the very few groups to have addressed this issue. The focus of their model was to consider how cost effective it is to treat IDU patients with HCV on a maintenance program, address issues regarding the implications of making treatment available to all IDU patients who meet the criteria and consider the effects on cost effectiveness if the age of stabilization of drug therapy lowered and also to assess the variation of cost effectiveness among Maori subgroups. The analysis compared dual interferon and ribavirin therapy with no antiviral therapy in New Zealand. A Markov model was constructed and adapted to include a higher dropout rate of $50 \%{ }^{2}$ compared with that of $20 \%-30 \%$ in the studies of Manns ${ }^{43}$ and Fried. ${ }^{42}$ Assumptions for the model came from data in which the community acquired rate of progression to chronic hepatitis $\mathrm{C}$ is an estimated $8 \%$, much less than the $20 \%$ used in other models. The model simulated a chronic relapsing pattern before stabilization of the patient on methadone replacement at the age of approximately 31 years of age. Costs included treatment of drug abuse and hepatitis $\mathrm{C}$. The costs for methadone replacement therapy were taken from previous costs within New Zealand. The cost of non-pegylated interferon plus ribavirin therapy was taken from Australian counterparts, as it was not offered under the New Zealand health system, with the assumed cost of pegylated interferon being set at 20\% higher than its non-pegylated alternative. These costs were direct, in keeping with assessment from a health institution point of view. Sensitivity analysis varied rates of progression of the disease. Benefits were recorded in life years, and data were presented in terms of incremental costs per life year gained. No QOL analysis was performed. Overall, both interferon and peginterferon remained cost effective compared with no treatment. Incremental cost-effectiveness ratios, measured in cost per life year gain, were favorable compared with no treatment. This was very much the case in non-Maori subgroups. The study also found that reducing the age of stabilization from the average of 31 to 26 years of age would be even more cost effective.

Much of the data upon which the cost-effectiveness analyses are based assumes compliance, and this is to be expected to some degree within the supported environment of controlled trials. We can speculate that the participants in the trials were likely to be highly motivated and adherent to treatment courses, and yet even with this assumption $20 \%-30 \%$ of participants failed to complete the course of treatment due to adverse effects. While Sheerin and colleagues ${ }^{66}$ demonstrated that patients who were stabilized on substance misuse therapy and also managed for HCV remained a cost-effective option, it is unclear how many of this vulnerable group are even referred or enrolled for treatment. Initial reports of case series of treatment outcomes showed much lower rates of SVR were achieved compared with clinical trials. However, more recent reports show rates comparable to and exceeding those seen in clinical trials, 
particularly with the most recent cohorts. ${ }^{67}$ This appears to be due to the evolution of better pathways of care, offering patient support to adhere to therapy, and more conservative therapy reductions to deal with side effects. The additional costs of these supports need to be factored into the analysis especially of some of these more difficult to treat patient groups, but this is likely to be balanced with much lower dropout rates than were modeled in the analysis. Another factor that needs to be considered when interpreting these analyses is the assumption of normal survival post SVR. Hopefully this is a valid assumption for most patients; it is less certain for those who are already cirrhotic, although undoubtedly better than if they had ongoing infection. The survival of patients on methadone treatment programs for opiate dependence is reduced, ${ }^{68}$ and this will reduce the cost effectiveness of anti-HCV treatment in this patient group, as it will reduce their post cure survival.

\section{Future developments}

There are two major developments occurring in the field of HCV therapy that are yet to undergo detailed cost-effectiveness analysis: reduced treatment courses guided by patient response and the development of new drugs. The reduced treatment courses are already approved for patients with G1, with low viral loads, who become HCV PCR negative after 4 weeks of therapy, a rapid virological response (RVR). In these patients, treatment can be reduced from 48 weeks to 24 weeks. Patients in this group have high SVR rates, greater than $80 \% .{ }^{69}$ This subgroup of patients represents about $25 \%-30 \%$ of most G1 cohorts in European countries. It does not require formal analysis to conclude that this will enhance the cost effectiveness of therapy. Similar reduced duration regimes based on an RVR are being developed for genotypes 2 and 3. Optimal duration of therapy is yet to be fully defined but ranges between 12 and 16 weeks. ${ }^{70}$

There are a significant number of new therapies for $\mathrm{HCV}$ in development. Most of these are protease inhibitors directed against G1 virus. Other classes of drug are also being developed but are not so close to licensing. Two new protease inhibitors are likely to be licensed for use in 2011 or soon after: telaprivir and boceprivir. ${ }^{71,72}$ These two drugs are both used for part of the treatment period and increase SVR in both treatment naïve and experienced patients. They may also reduce treatment duration. There is no indication of the cost of these products, but they are likely to be expensive and will require careful evaluation of their cost effectiveness. If they are priced too high, it may be more cost effective to treat patients with good predictors of response to therapy with current standard of care and reserve the new drugs for treatment failures and those with poor predictors of response.

\section{Conclusions}

It can be concluded that dual therapy with pegylated interferon and ribavirin remains the treatment of choice for $\mathrm{HCV}$ infection, and this choice is cost effective for use in most health care systems. The use of patient-specific early viral responses will prevent further adverse effects of continued treatment with little or no added benefit to the patient population and also allow shortened therapy for some with no adverse effect on outcome. The key to maintaining the cost effectiveness of therapy is the SVR rate. If this falls too far from those rates seen in the RCT's used to inform the cost-effectiveness models, then the therapy may no longer be cost effective. The use of adjuncts to treatment, like hematopoietic growth factors, may improve patient adherence with treatment and QOL, but have yet to be shown to improve SVR; and unless the size of this improvement is large they are unlikely to be cost effective in most health care settings. The costs of bringing patients from more difficult to treat backgrounds into therapy and maintaining them in treatment have up until now not been addressed in any cost-effectiveness analysis, and as the treatment population is increasingly dominated by this group, further analysis will be needed to take this into account. While there is no clear evidence that starting HCV treatment will cause people from an IVDU background to change health behavior and enter or stabilize on an opiate substitution program, it is another health benefit that early reports are suggesting might be relevant and if substantiated would increase the cost effectiveness of therapy in this group. There is clear evidence that treatment of $\mathrm{HCV}$ is cost effective, but this is a complex and rapidly evolving treatment area and much of the analysis reviewed will quickly be outdated by application of treatment to different disease populations and the arrival of new drugs.

\section{Disclosure}

The authors report no conflicts of interest in this work.

\section{References}

1. Mühlberger NSR, Lettmeier B, Sroczynski G, Zeuzem S, Siebert U. HCVrelated burden of disease in Europe: a systematic assessment of incidence, prevalence m, and mortality. BMC Public Health. 2009 Jan 22;9:34, PMC2656539. RPPPCP

2. WHO. HEPATITIS C. 2002;http://www.who.int/csr/disease/hepatitis/ Hepc.pdf

3. Health Protection Agency (Hepatitis C in UK). http://wwwhpaorguk/ hepC2009 
4. Scotland HP. Hepatitis C in Scotland http://wwwdocumentshpsscotnhsuk/ ewr/pdf2009/0918pdf

5. Patel HHE. When to treat and the benefits of treating hepatitis $\mathrm{C}$ in patients with haemophilia. Haemophilia. 2009;15(1):20-32.

6. Alter HJPR, Shih JW, Melpolder JC, Houghton M, Choo QL, Kuo G. Detection of antibody to hepatitis $\mathrm{C}$ virus in prospectively followed transfusion recipients with acute and chronic non-A, non-B hepatitis. N Engl J Med. 1989 Nov 30;321(22):1494-1500.

7. Poynard T, Bedossa P, Opolon P. Natural history of liver fibrosis progression in patients with chronic hepatitis C. The Lancet. 1997;349(9055): 825-832.

8. Mattsson LSA, Weiland O. Outcome of acute symptomatic non-A, non-B hepatitis: a 13-year follow-up study of hepatitis $\mathrm{C}$ virus markers. Liver. 1993;13;274-278.

9. Tremolada FCC, Alberti A, Drago C, Tagger A, Ribero ML, Realdi G. Long-term follow-up of non-A, non-B (type C) post-transfusion hepatitis. J Hepatol. 1992 Nov;16(3):273-281.

10. Di Bisceglie AMGZ, Ishak KG, Hoofnagle JH, Melpolder JJ, Alter HJ. Long-term clinical and histopathological follow-up of chronic posttransfusion hepatitis. Hepatology. 1991 Dec;14(6):969-974.

11. Koretz RLAH, Coleman E, Gitnick G. Non-A, non-B post-transfusion hepatitis - looking back in the second decade. Ann Intern Med. 1993; 119:110-115.

12. Tong MJ, El-Farra NS, Reikes AR, Co RL. Clinical Outcomes after Transfusion-Associated Hepatitis C. N Engl J Med. 1995 June 1; 332(22):1463-1466.

13. Yano M, Kumada H, Kage M, Ikeda K, Shimamatsu K, Inoue O, et al. The long-term pathological evolution of chronic hepatitis C. Hepatology. 1996;23(6):1334-1340.

14. Kiyosawa KST, Tanaka E, Gibo Y, et al. Interrelationship of blood transfusion, non-A, non-B hepatitis and hepatocellular carcinoma: Analysis by detection of antibody to hepatitis C virus. Hepatology. 1990;12(4): 671-675.

15. Niederau CLS, Heintges T, Erhardt A, Buschkamp M, Hürter D, Nawrocki M, Kruska L HF, Petry W, Häussinger D. Prognosis of chronic hepatitis c: Results of a large, prospective cohort study. Hepatology. 1998;28(6):1687-1695.

16. Vogt M, Lang T, Frosner G, Klingler C, Sendl AF, Zeller A, et al. Prevalence and Clinical Outcome of Hepatitis C Infection in Children Who Underwent Cardiac Surgery before the Implementation of Blood-Donor Screening. N Engl J Med. 1999 Sep 16;341(12): 866-870.

17. Seeff LBHF, Alter HJ, Wright EC, et al. Long-term mortality and morbidity of transfusion-associated non-A, non-B, and type $\mathrm{C}$ hepatitis: A National Heart, Lung, and Blood Institute collaborative study. Hepatology. 2001;33(2):455-463.

18. Seeff LB, Miller RN, Rabkin CS, Buskell-Bales Z, Straley-Eason KD, Smoak BL, et al. 45-Year Follow-up of Hepatitis C Virus Infection in Healthy Young Adults. Annals of Internal Medicine. 2000 Jan 18;132(2):105-111.

19. Seeff LB. The history of the "natural history" of hepatitis C (1968-2009). Liver International. 2009;29(s1):89-99.

20. Sterling RK, Stravitz RT, Luketic VA, Sanyal AJ, Contos MJ, Mills AS, et al. A comparison of the spectrum of chronic hepatitis $\mathrm{C}$ virus between Caucasians and African Americans. Clinical Gastroenterology and Hepatology. 2004;2(6):469-473.

21. Hutchinson SJ, Bird SM, Goldberg DJ. Influence of Alcohol on the Progression of Hepatitis C Virus Infection: A Meta-analysis. Clinical Gastroenterology and Hepatology. 2005;3(11):1150-1159.

22. Westin J, Lagging LM, Spak F, Aires N, Svensson E, Lindh M, et al. Moderate alcohol intake increases fibrosis progression in untreated patients with hepatitis $\mathrm{C}$ virus infection. Journal of Viral Hepatitis. 2002;9(3):235-241.

23. Adinolfi LEGM, Andreana A, Tripodi MF, Utili R, Ruggiero G. Steatosis accelerates the progression of liver damage of chronic hepatitis $\mathrm{C}$ patients and correlates with specific HCV genotype and visceral obesity. Hepatology. 2001;33(6):1358-1364.
24. Bica I, McGovern B, Dhar R, Stone D, McGowan K, Scheib R, et al. Increasing Mortality Due to Endâ€ Stage Liver Disease in Patients with Human Immunodeficiency Virus Infection. Clinical Infectious Diseases. 2001;32(3):492-427.

25. Sulkowski MSMS, Torbenson MS, Higgins Y, et al. Rapid fibrosis progression among HIV/hepatitis C virus-co-infected adults. AIDS. 2007;21(16):2209-2216.

26. Fattovich G, Giustina G, Degos F, Tremolada F, Diodati G, Almasio P, et al. Morbidity and mortality in compensated cirrhosis type C: A retrospective follow-up study of 384 patients. Gastroenterology. 1997;112(2): 463-472.

27. Kim WR. The burden of hepatitis $\mathrm{C}$ in the United States. Hepatology. 2002;36(5B):s30-s4.

28. Foster GR, Goldin RD, Thomas HC. Chronic hepatitis C virus infection causes a significant reduction in quality of life in the absence of cirrhosis. Hepatology. 1998;27(1):209-212.

29. SIGN. Managment of Hepatitis C http://www.sign.ac.uk/pdf/sign92. pdf. 2006.

30. NICE. Hepatitis C http://www.nice.org.uk/TA106. 2006.

31. EASL International Consensus Conference on Hepatitis C Paris, 1999 Feb 26-28: Consensus Statement*. Journal of Hepatology. 1999;30(5): 956-961.

32. Edlin BR, Seal KH, Lorvick J, Kral AH, Ciccarone DH, Moore LD, et al. Is It Justifiable to Withhold Treatment for Hepatitis $\mathrm{C}$ from IllicitDrug Users? N Engl J Med. 2001 Jul 19;345(3):211-214.

33. Chang CH, Chen KY, Lai MY, Chan KA. Meta-analysis: ribavirininduced haemolytic anaemia in patients with chronic hepatitis C. Alimentary Pharmacology and Therapeutics. 2002;16(9): 1623-1632.

34. Dieterich DT, Wasserman R, Brau N, Hassanein TI, Bini EJ, Bowers PJ, et al. Once-weekly epoetin alfa improves anemia and facilitates maintenance of ribavirin dosing in hepatitis $\mathrm{C}$ virus-infected patients receiving ribavirin plus interferon alfa. Am J Gastroenterol. 2003;98(11):2491-2499.

35. Pockros PJSM, Schiff ER, Sulkowski MS, et al. Proactive Study Group. Epoetin alfa improves quality of life in anemic HCV-infected patients receiving combination therapy. Hepatology. 2004;40(6): 1450-1458.

36. Muir AJMJ. Growth factors during HCV therapy may be "cost-effective", but are they "effective"? Hepatology. 2006;44(6):1400-1403.

37. McHutchison JGGS, Schiff ER, et al Interferon alfa-2b alone or in combination with ribavirin as initial treatment for chronic hepatitis C. New Eng J Med. 1998:339:1485-1492.

38. Poynard TMP, Lee SS, et al. Randomised trial of interferon $\alpha 2 b$ plus ribavirin for 48 weeks or for 24 weeks versus interferon $\alpha 2 b$ plus placebo for 48 weeks for treatment of chronic infection with hepatitis C virus. Lancet. 1998;352:1426-1432.

39. Kjaegard LL KK, Gluud C. Interferon alfa with or without ribavirin for chronic hepatitis C: Systematic review of randomised trial. BMJ. 2001; 323:1151-1155.

40. Luxon BA, Grace M, Brassard D, Bordens R. Pegylated interferons for the treatment of chronic hepatitis $\mathrm{C}$ infection. Clinical Therapeutics. 2002;24(9):1363-1383.

41. Foster GR. Pegylated Interferons for the Treatment of Chronic Hepatitis C: Pharmacological and Clinical Differences between Peginterferon-[alpha]-2a and Peginterferon-[alpha]-2b. Drugs. 70(2): 147-165.

42. Fried MW, Shiffman ML, Reddy KR, Smith C, Marinos G, Goncales FL Jr, et al. Peginterferon Alfa-2a plus Ribavirin for Chronic Hepatitis C Virus Infection. N Engl J Med. 2002 Sep 26;347(13): 975-982.

43. Manns MP, McHutchison JG, Gordon SC, Rustgi VK, Shiffman M, Reindollar R, et al. Peginterferon alfa-2b plus ribavirin compared with interferon alfa-2b plus ribavirin for initial treatment of chronic hepatitis $\mathrm{C}$ : a randomised trial. The Lancet. 2001;358(9286):958-965.

44. Davis G. Monitoring of viral levels during therapy of hepatitis C. Hepatology. 2002;36(5B):s145-s51. 
45. Hadziyannis SJ, Sette H, Morgan TR, Balan V, Diago M, Marcellin $\mathrm{P}$, et al. Peginterferon-ÎI $+2 \mathrm{a}$ and Ribavirin Combination Therapy in Chronic Hepatitis C. Annals of Internal Medicine. 2004 Mar 2;140(5): 346-355.

46. Taylor LE, Rich JD, Tashima KT, Gholam PM, Torriani FJ, Dieterich DT, et al. Peginterferon plus Ribavirin for Hepatitis C in HIV-Infected Patients. N Engl J Med. 2004 Nov 25;351(22):2340-2342.

47. Carrat F, Bani-Sadr F, Pol S, Rosenthal E, Lunel-Fabiani F, Benzekri A, et al. Pegylated Interferon Alfa-2b vs Standard Interferon Alfa-2b, Plus Ribavirin, for Chronic Hepatitis C in HIV-Infected Patients: A Randomized Controlled Trial. JAMA. 2004 Dec15;292(23):2839-2848.

48. McHutchison JG, Manns M, Patel K, Poynard T, Lindsay KL, Trepo C, et al. Adherence to combination therapy enhances sustained response in genotype-1-infected patients with chronic hepatitis C. Gastroenterology. 2002;123(4):1061-1069.

49. McHutchison J, Sulkowski M. Scientific rationale and study design of the individualized dosing efficacy vs flat dosing to assess optimal pegylated interferon therapy (IDEAL) trial: determining optimal dosing in patients with genotype 1 chronic hepatitis C. Journal of Viral Hepatitis. 2008;15(7):475-481.

50. Awad T TK, Hauser G, Stimac D, Mabrouk M, Gluud C. Peginterferon alpha-2a is associated with higher sustained virological response than peginterferon alfa- $2 b$ in chronic hepatitis $C$ : Systematic review of randomized trials. Hepatology. 51(4):1176-1184.

51. Poynard T, Cacoub P, Ratziu V, Myers RP, Dezailles MH, Mercadier A, et al. Fatigue in patients with chronic hepatitis C. Journal of Viral Hepatitis. 2002;9(4):295-303.

52. Ware JE Jr BM, Mannocchia M, Davis GL, International Hepatitis Interventional Therapy G. Health-related quality of life in chronic hepatitis C: Impact of disease and treatment response. Hepatology. 1999;30(2):550-555.

53. Younossi ZM, Guyatt G, Kiwi M, Boparai N, King D. Development of a disease specific questionnaire to measure health related quality of life in patients with chronic liver disease. Gut. 1999 Aug 1;45(2):295-300.

54. Younossi ZKJ, Kincaid J. The effects of HCV infection and management on health-related quality of life. Hepatology. 2007;45(3):806-816.

55. Bonkovsky HLWJ. Group CIS. Reduction of health-related quality of life in chronic hepatitis $\mathrm{C}$ and improvement with interferon therapy. Hepatology. 1999;29(1):264-270.

56. Carithers RL Jr SD, Bayliss M. Health assessment for chronic HCV infection: results of quality of life. Dig Dis Sci. 1996 Dec;41 Suppl 12:75S-80S. PubMed PMID.

57. Forton DMTH, Murphy CA, Allsop JM, Foster GR, Main J, Wesnes KA, SDTR. Hepatitis C and cognitive impairment in a cohort of patients with mild liver disease. Hepatology. 2002;35(2):433-439.

58. Neary MPCS, Bayliss MS, Ware JE Jr. Sustained virologic response is associated with improved health-related quality of life in relapsed chronic hepatitis C patients. Semin Liver Dis. 1999;19 Suppl 1:77-85.

59. Fontana RJ, Hussain KB, Schwartz SM, Moyer CA, Su GL, Lok ASF. Emotional distress in chronic hepatitis $\mathrm{C}$ patients not receiving antiviral therapy. Journal of Hepatology. 2002;36(3):401-407.
60. Negro F. Adverse effects of drugs in the treatment of viral hepatitis. Best Practice and Research Clinical Gastroenterology. 24(2):183-192.

61. Bernfort L, SennfÃ €lt K, Reichard O. Cost-effectiveness of peginterferon alfa-2b in combination with ribavirin as initial treatment for chronic hepatitis C in Sweden. Scandinavian Journal of Infectious Diseases. 2006;38(6-7):497-505.

62. Siebert U, Sroczynski G, Rossol S, Wasem J, Ravens-Sieberer U, Kurth $\mathrm{BM}$, et al. Cost effectiveness of peginterferon $\hat{\mathrm{I}}+-2 \mathrm{~b}$ plus ribavirin versus interferon $\hat{\mathrm{I}} \pm-2 \mathrm{~b}$ plus ribavirin for initial treatment of chronic hepatitis C. Gut. 2003 Mar;52(3):425-432.

63. Wong JB, Davis GL, Pauker SG. Cost effectiveness of ribavirin/ interferon alfa- $2 \mathrm{~b}$ after interferon relapse in chronic hepatitis $\mathrm{C}$. The American Journal of Medicine. 2000;108(5):366-373.

64. Buti M, Medina M, Casado MA, Wong JB, Fosbrook L, Esteban R. A cost-effectiveness analysis of peginterferon alfa- $2 b$ plus ribavirin for the treatment of naive patients with chronic hepatitis C. Alimentary Pharmacology and Therapeutics. 2003;17(5):687-694.

65. Shepherd JBH, Cave CB, Waugh NR, Price A, Gabbay J. Clinical- and cost-effectiveness of pegylated interferon alfa in the treatment of chronic hepatitis C: a systematic review and economic evaluation. Int JTechnol Assess Health Care. 2005 Winter:21(1):47-54.

66. Sheerin IG, Green FT, Sellman JD. What is the cost-effectiveness of hepatitis $\mathrm{C}$ treatment for injecting drug users on methadone maintenance in New Zealand? Drug and Alcohol Review. 2004;23(3):261-272.

67. Grishchenko M, Grieve RD, Sweeting MJ, de Angelis D, Thomson BJ, Ryder SD, et al. Cost-effectiveness of pegylated interferon and ribavirin for patients with chronic hepatitis $\mathrm{C}$ treated in routine clinical practice. International Journal of Technology Assessment in Health Care. 2009;25(02):171-180.

68. McCowan C, Kidd B, Fahey T. Factors associated with mortality in Scottish patients receiving methadone in primary care: retrospective cohort study. BMJ. 2009 Jun 16;338(jun16_4):b2225.

69. Zeuzem SPJ, Lukasiewicz E, et al. DITTO-HCV Study Group. International, multicenter, randomized, controlled study comparing dynamically individualized versus standard treatment in patients with chronic hepatitis C. J Hepatol. 2005 Aug;43(2):250-257.

70. Diago MSM, Bronowicki JP, Zeuzem S, Rodriguez-Torres M, Pappas SC, Tietz AND. Identifying hepatitis $C$ virus genotype $2 / 3$ patients who can receive a 16 -week abbreviated course of peginterferon alfa-2a (40KD) plus ribavirin. Hepatology. 9999(9999):NA.

71. Berman K, Kwo PY. Boceprevir an NS3 Protease Inhibitor of HCV. Clinics in Liver Disease. 2009;13(3):429-439.

72. McHutchison JGEG, Gordon SC, Jacobson IM, et al. PROVE1 Study Team. Telaprevir with Peginterferon and Ribavirin for Chronic HCV Genotype 1 Infection. N Engl J Med. 2009 Oct 8;361(15):1516.
ClinicoEconomics and Outcomes Research

\section{Publish your work in this journal}

ClinicoEconomics \& Outcomes Research is an international, peerreviewed open-access journal focusing on Health Technology Assessment, Pharmacoeconomics and Outcomes Research in the areas of diagnosis, medical devices, and clinical, surgical and pharmacological intervention. The economic impact of health policy and health systems

\section{Dovepress}

organization also constitute important areas of coverage. The manuscript management system is completely online and includes a very quick and fair peer-review system, which is all easy to use. Visit $\mathrm{http}: / /$ www.dovepress.com/testimonials.php to read real quotes from published authors. 